\title{
Putting hemispheric asymmetry to use in understanding brain diseases
}

\author{
Harold W. Gordon* \\ Epidemiology Research Branch, Division of Epidemiology, Services, and Prevention Research, National Institute on Drug Abuse, 6001 Executive Boulevard, \\ Room 5151, Bethesda, MD 20893, USA
}

The time has come to use what we have learned over the past century and a half about hemispheric asymmetries to understand the bases of brain diseases. Left/right differences have been described for cognitive function, regional brain activation, neuronal connectivity, cortical thickness, structural volume, and neurotransmitter concentration in healthy individuals as well as for those with different forms of mental dysfunction. The missing element in research is to use these hemispheric differences to develop hypotheses to understand the underlying causes of the disorders. It is not enough, for example, to describe in schizophrenic patients language deficits which are attributable to the left hemisphere. Or suggest that impulsivity-a function controlled by the right hemisphere-is a problem for individuals with Attention Deficit Disorder or addiction. The question is whether there is anything about the neurobiology of the left and right hemispheres that we can use to give insight to the causes of these brain diseases. Today's technology to understand the brain at the cellular level can now be combined with what we know about cognitive behaviors to develop appropriate hypotheses.

The most oft-repeated hemispheric asymmetry associated with a mental illness is left hemisphere dysfunction or abnormality in patients with schizophrenia. This includes functional reduction for language [1], abnormal asymmetry for gray matter volume and functional activity in the temporal lobe [2], and interhemispheric connectivity [3]. But how can we use these observations to better understand the basis of the schizophrenic disorder? Conclusions from a recent review [4] were one step in the correct direction by taking symptomatology into account together with hemispheric function. Here, it was observed that patients with an "active" syndrome were more affected by dysfunction in the left hemisphere; those with a withdrawn or negative syndrome were more affected by dysfunctions in the right hemisphere. These dysfunctions included several neurophysiological and behavioral measures. A similar distinction was made between patients presenting with a constellation of symptoms of "positive" (hyperactivity, grandiosity, hostility, suspiciousness, delusion, hallucinations) or "negative" (blunted affect, emotional withdrawal, apathy, stereotypy or difficulty in thinking, lack of spontaneity) nature [5]. Patients in the "positive" symptom group had greater dysfunctions in the left hemisphere; those in the "negative" group had greater dysfunctions in the right hemisphere. The conclusions for both these studies are reminiscent of the well-known (in the psychological literature) of the left/right hemispheric differences for approach/avoidance behavior, respectively. These constructs have a research track record of activation asymmetries and neurotransmitter asymmetries. The strategy now should be to meld these different fields to develop hypotheses leading to understanding and treatment of, in this case, schizophrenia.
The approach/avoidance dichotomy also plays a role in patients with Attention Deficit Hyperactive Disorder (ADHD). Whether the behavior in this disorder is considered to be an aggressive approach or a failure of inhibition, a more active left hemisphere or a less active right hemisphere would be consistent with this observation. Accordingly, two studies demonstrate this. One study showed a more active left hemisphere in the resting state [6] while another study showed a failure of neuronal development in the right hemisphere [7]. Precisely the opposite was found in patients with Obsessive-Compulsive Disorder (OCD) where there was greater activation in the right hemisphere [8]. In keeping with the approach/avoidance dichotomy, this affirms the face validity aspect where OCD patients have a greater tendency for avoidance. But another study [9] showed increased thickness of the anterior cingulate cortex of the left hemisphere in OCD patients as well as their siblings. As with schizophrenia, the next step would be to use these observations of asymmetry (and the underlying neurobiology) to develop hypotheses focused on the underlying neurobiological causes of the disorders. An early study in OCD patients attempted to do this by observing that there was greater binding for dopamine transporters in the left basal ganglia [10]. Clearly these differential asymmetry reports need to be reconciled by a team of researchers representing multiple disciplines. The point here is that hemispheric asymmetries seem to be real and, with greater focus on them, they can better inform etiologies of these disorders.

Important places to look for underlying causes of adverse symptomatology are in the systems of neuronal activation, connectivity and neurotransmitter circuitry. In a recent survey to determine brain activation in behaviors related to drug abuse, I compiled lateral, cue-activated brain responses for impulsivity-a risk factor for drug abuse-and craving. There were more instances across studies of right hemisphere activation for measures of impulsivity and left hemisphere activation for cue-induced craving [11]. These differential left/ right activation effects coincide nicely with the avoidance/approach dichotomy found for other brain diseases mentioned above. Again, these observations should be helpful in understanding the underlying etiology of drug abuse. Hemispheric laterality is just as important for studying drug abuse as it appears to be for psychiatric disorders.

Correspondence to: Harold W. Gordon, Epidemiology Research Branch, Division of Epidemiology, Services, and Prevention Research, National Institute on Drug Abuse, 6001 Executive Boulevard, Room 5151, Bethesda, MD 20893, Tel: +1 301 443-6504, USA, E-mail: hg23r@nih.gov

Received: October 28, 2016; Accepted: December 06, 2016; Published: December 10, 2016 
Can we learn anything about the underlying neurobiology? Perhaps so, by determining what effect the drugs themselves have on morphological development and functional activation of regions of the brain. To answer this question, I compiled data from research reports that described which brain structures were affected when exposed to the different drugs of abuse (presented at the Annual Meeting of the Society for Neuroscience, San Diego, November, 2016). Two surveys were conducted: one in which brain measures (primarily cortical thickness or gray matter volume) were made in individuals who had been prenatally exposed to drugs of abuse; another in individuals who became drug dependent either as adolescents or adults. Among the results, cocaine is the most illustrative. For both groups of subjectsthose exposed prenatally or as adolescents/adults-affected structures were mentioned more often in either the left or right hemisphere rather than bilaterally. Furthermore, the unilateral effects were not equally distributed between the left and right hemispheres. For individuals who were exposed to cocaine prenatally, more structures were affected in the left hemisphere; by contrast, more structures were affected in the right hemisphere for individuals who had adolescent- or adultonset dependence. The switch in distribution was significant $(\mathrm{p}<$ .05 ). By contrast, exposure to alcohol had the opposite effect: more structures were affected in the right hemisphere for prenatal exposure and equally in both for adult-onset dependence. For nicotine there was no significant difference affecting one hemisphere more than the other for either prenatal or adolescent/adult exposure. When exposed to different exogenous substances, what does this tell us about neuronal development? More research could help elucidate these questions.

We have come full circle from the studies of lateralization of cognitive function dating from the 1960's together with psychological constructs such as approach/avoidance that map to the left/right hemisphere dichotomy, and extending to laterality research with advanced imaging techniques. Finally, data are emerging in each of several disciplines for healthy as well as abnormally-developed brains that can now provide clues to underlying causes of mental disorders. For example, hemispheric asymmetries are related to differential concentrations and activations of neurotransmitter systems, notably dopamine. All that is needed now is to heed conclusions from the variety of neurobiological studies of hemispheric asymmetry and gather research teams with the variety of expertise in neuropsychology, neurophysiology, and neuroimaging to generate hypotheses of what the laterality data are telling us about brain disorders including those of drug abuse. We can no longer live in our silos and feel comfortable to only report our observations. The time is now to put our collective knowledge together, generate hypotheses and move up to the next level of research to understand the neurobiological bases so that prevention and intervention strategies can be developed.

\section{Disclaimer}

The views and opinions expressed in this manuscript are those of the author only and do not necessarily represent the views, official policy or position of the U.S. Department of Health and Human Services or any of its affiliated institutions or agencies.

\section{References}

1. Royer C, Delcroix N, Leroux E, Alary M, Razafimandimby A, et al. (2015) Functional and structural brain asymmetries in patients with schizophrenia and bipolar disorders. Schizophr Res 161: 210-214. [Crossref]

2. Oertel V, Knöchel C, Rotarska-Jagiela A, Schönmeyer R, Lindner M, et al. (2010) Reduced laterality as a trait marker of schizophrenia--evidence from structural and functional neuroimaging. $J$ Neurosci 30: 2289-2299. [Crossref]

3. Ribolsi M, Daskalakis ZJ, Siracusano A, Koch G (2014) Abnormal asymmetry of brain connectivity in schizophrenia. Front Hum Neurosci 8: 1010. [Crossref]

4. Gruzelier JH (1999) Functional neuropsychophysiological asymmetry in schizophrenia a review and reorientation. Schizophr Bull 25: 91-120. [Crossref]

5. Ke M, Zou R, Shen H, Huang X, Zhou Z, et al. (2010) Bilateral functional asymmetry disparity in positive and negative schizophrenia revealed by resting-state fMRI. Psychiat Res: Neuroimaging 182: 30-39. [Crossref]

6. Keune PM, Schönenberg M, Wyckoff S, Mayer K, Riemann S, et al. (2011) Frontal alpha-asymmetry in adults with attention deficit hyperactivity disorder: Replication and specification. Biol Psychol 87: 306-310. [Crossref]

7. Shaw P, Lalonde F, Lepage C, Rabin C, Eckstrand K, et al. (2009) Development of cortical asymmetry in typically developing children and its disruption in AttentionDeficit/Hyperactivity Disorder. Arch Gen Psychiatry 66: 888-896. [Crossref]

8. Ischebeck M, Endrass T, Simon D, Kathmann N (2014) Altered frontal EEG asymmetry in obsessive-compulsive disorder. Psychophysiology 51: 596-601. [Crossref]

9. Peng Z, Li G, Shi F, Shi C, Yang Q, et al. (2015) Cortical asymmetries in unaffected siblings of patients with obsessive-compulsive disorder. Psychiatry Res 234: 346-351. [Crossref]

10. van der Wee NJ, Stevens H, Hardeman JA, Mandl RC, Denys DA, et al. (2004) Enhanced dopamine transporter density in psychotropic-naive patients with ObsessiveCompulsive Disorder shown by [123I] \{beta\}-CIT SPECT. Am J Psychiat 161: 22012206. [Crossref]

11. Gordon HW (2016) Laterality of Brain Activation for Risk Factors of Addiction. Cur Drug Abuse Rev 9: 1-18. [Crossref]

Copyright: (C2016 Gordon HW. This is an open-access article distributed under the terms of the Creative Commons Attribution License, which permits unrestricted use, distribution, and reproduction in any medium, provided the original author and source are credited. 